\title{
PREVALENCIA DE MICROORGANISMOS ASOCIADOS A INFECCIONES VAGINALES EN 230 MUJERES GESTANTES Y NO GESTANTES SINTOMÁTICAS DEL CENTRO DE SALUD LA MILAGROSA EN EL MUNICIPIO DE ARMENIA (COLOMBIA)
}

\author{
Prevalence of micro-organisms associated with \\ vaginal infections in 230 symptomatic expectant \\ and non-expectant women at La Milagrosa health \\ center in Armenia, Colombia \\ Natalia Salas, Biol.*, Juan Felipe Ramírez, Biol.*, Bayron Ruiz, M.D.**, \\ Elizabeth Torres, Bact.***, Luis Nevio Jaramillo, Bact.****, \\ Jorge Enrique Gómez-Marín, M.D.** \\ Recibido: febrero 28/09 - Aceptado: abril 3/09
}

\section{RESUMEN}

Objetivos: determinar la prevalencia de los agentes etiológicos de las infecciones vaginales en mujeres embarazadas y no embarazadas sintomáticas del centro de salud "La Milagrosa", de Armenia (Quindío, Colombia), en un periodo comprendido entre noviembre y diciembre de 2007 y enero de 2008.

Materiales y métodos: estudio de prevalencia. Se tomaron muestras en 230 pacientes que consultaron

* Biólogo. Grupo de Estudio en Parasitología Molecular (GEPAMOL). Centro de Investigaciones Biomédicas, Universidad del Quindío. Armenia (Colombia).

** M.D. Grupo de Estudio en Parasitología Molecular (GEPAMOL). Centro de Investigaciones Biomédicas, Universidad del Quindío. Armenia (Colombia). Correspondencia: Jorge Enrique Gómez Marín, Centro de investigaciones biomédicas, Universidad del Quindío, Av. Bolívar 12N, Armenia (Quindío, Colombia). Tel/Fax: (57)6 7460168. Correo electrónico: gepamol2@ uniquindío.edu.co

*** Bacteriólogo. Grupo de Estudio en Parasitología Molecular (GEPAMOL). Centro de Investigaciones Biomédicas, Universidad del Quindío. Armenia (Colombia).

**** Bacteriólogo. Grupo de Estudio en Parasitología Molecular (GEPAMOL). Centro de Investigaciones Biomédicas, Universidad del Quindío. Armenia (Colombia). por síntomas asociados a infección vaginal en el centro de salud "La Milagrosa". A todas las pacientes se les tomó muestras de flujo vaginal para la medición del $\mathrm{pH}$, test de amina, identificación microscópica de células clave (células epiteliales que contienen bacterias, indicando la presencia de Gardnerella), Trichomonas vaginalis, levaduras e hifas. Se hicieron cultivos en agar sangre, Sabouraud y Mac Conckey y se realizó la técnica de tinción de Gram. Los datos fueron analizados en el programa Epi Info versión 6.

Resultados: la principal causa de infección fue cocobacilos gram variable tipo Gardnerella (39\%), seguida de Candida spp (6,5\%) y Trichomonas vaginalis $(5,7 \%)$.

Conclusiones: en pacientes sintomáticas de flujo vaginal se encontró mayor prevalencia de vaginosis bacteriana.

Palabras clave: Gardnerella, candidiasis, tricomoniasis, flujo, prurito, infecciones mixtas. 


\section{SUMMARY}

Objective: determining the prevalence of infectious agents responsible for vaginal infections in symptomatic women at La Milagrosa primary health center in Armenia (Quindío, Colombia), between November and December 2007 and January 2008.

Materials and methods: a cross sectional study was carried out on 230 patients who consulted for vaginal discharge and symptoms. A sample of vaginal discharge was obtained from each patient. Each sample was analysed for $\mathrm{pH}$, amines test, identification of clue cells containing bacteria as an indicator for Gardnerella infection, Trichomonas vaginalis and the presence of hifae or yeasts. All samples were also cultured on blood agar, Sabouraud and Mac Conckey media and Gram stained. The data was organised on an Excel spreadsheet and analysed using Epi Info software (version 6).

Results: Gardnerella had the highest infectious agent prevalence (39\%), followed by Candida spp (6.5\%) and Trichomonas vaginalis (5.7\%).

Conclusions: Symptomatic patients with vaginal fluid presented a higher prevalence of bacterial vaginosis.

Key words: Gardnerella, candidiasis, trichmoniasis, vaginitis, vaginal discharge, bacterial vaginosis.

\section{INTRODUCCIÓN}

Las infecciones vaginales caracterizadas por flujo vaginal con síntomas como molestia o ardor en región vaginal, dolor pélvico o prurito, y mal olor genital son una de las causas más frecuentes de consulta ginecológica en las mujeres en edad fértil. En estudios realizados a nivel mundial se ha encontrado que el 90\% de esas infecciones son causadas por tres grupos de agentes etiológicos: i) un grupo de bacterias anaerobias que producen vaginosis, ii) levaduras del género Candida spp y iii) infección por el parásito protozoario Trichomonas vaginalis. ${ }^{1} \mathrm{De}$ éstas, la vaginosis bacteriana es la más frecuente y tiene como agentes causales bacterias que sustituyen la flora normal de Lactobacillus acidophilus, tales como Mobiluncus spp, Prevotella spp, Mycoplasma hominis o Gardnerella vaginalis. Esta última es la de mayor frecuencia entre estos agentes bacterianos. ${ }^{2}$ Las prevalencias reportadas de vaginosis bacteriana son muy variables, y es así como se ha encontrado que han sido desde 5\% en estudiantes de secundaria hasta de $50 \%$ en zona rural, para un mismo país como Uganda. ${ }^{2}$

Existen dos maneras de realizar el diagnóstico de vaginosis. El primero de los dos métodos empleados se lleva a cabo de acuerdo con la presencia de, al menos, tres de cuatro criterios propuestos por Amsel y colegas ${ }^{3}$ y el otro se basa en el resultado de la tinción de Gram del flujo vaginal, aplicando los criterios planteados por Nugent. ${ }^{4}$ Los criterios de Amsel es el método más utilizado e incluye: $\mathrm{pH}$ mayor a 4,5, prueba de aminas positiva, descarga fina, blanca adherente y homogénea y presencia de células guía en el flujo vaginal (células epiteliales que contienen bacterias, indicando la presencia de Gardnerella). Cuando se encuentran presentes tres de estos criterios se concluye que se tiene entre 60 a $100 \%$ de sensibilidad. ${ }^{2}$

Otra de las infecciones vaginales más frecuentes es la producida por levaduras del género candida, principalmente Candida albicans. Los síntomas y signos característicos son: prurito vulvar, secreción espesa, eritema, dispareunia, disuria, una secreción blanca y el enrojecimiento de la mucosa vaginal y del epitelio vulvar. ${ }^{5}$ La tricomoniasis es también una infección vaginal común en mujeres que se encuentran en edad fértil y es la enfermedad de transmisión sexual más común, la cual se estima afecta a 170 millones de mujeres anualmente en todo el mundo. Esta infección, causada por Trichomonas vaginalis, un protozoo flagelado amitocondrial, produce sintomatología clínica similar a la de otros microorganismos como Candida spp y Gardnerella vaginalis. ${ }^{6}$

Los estudios epidemiológicos sobre la etiología de estas infecciones son necesarios con el fin de orientar los programas de control y prevención, las necesidades de investigación y dar herramientas al médico en la orientación del diagnóstico y el tratamiento. En una búsqueda bibliográfica con los términos "vaginitis" y "Colombia" en la base de datos PubMed, sólo se 
encontraron tres publicaciones, dos de ellas de la década de los 90 y una de 2004, sobre población que acudía a realizarse el examen de citología vaginal..$^{7-9}$ Otros estudios hallados en otras fuentes bibliográficas como Scielo, se llevaron a cabo en usuarias de planificación familiar, ${ }^{10}$ población carcelaria, ${ }^{11}$ población que se practicaba la citología cervical $^{12}$ y un estudio reciente en Montería en trabajadoras sexuales y 77 amas de casa como grupo control. ${ }^{13}$ Otro estudio internacional, específicamente sobre vaginosis, encontró $9 \%$ de prevalencia en pacientes colombianas asintomáticas. ${ }^{14} \mathrm{~A}$ diferencia de otros países latinoamericanos, no encontramos estudios colombianos en población sintomática. Por consiguiente, el objetivo del presente estudio fue determinar la prevalencia de agentes etiológicos de infección vaginal en la población de embarazadas y no embarazadas que asiste a consulta por síntomas relacionados, en el nivel primario y secundario de salud.

\section{MATERIALES Y MÉTODOS}

Este fue un estudio de prevalencia en el que se estudiaron mujeres en edad reproductiva (entre los 14 y los 45 años) que consultaron al centro de salud "La Milagrosa" de la ciudad de Armenia. Este centro de salud hace parte de la red pública de atención en salud, sirve a la población perteneciente al régimen de aseguramiento subsidiado (SISBEN 1 a 3) y realiza consulta ginecológica y obstétrica de primero y segundo nivel de complejidad. En el estudio se incluyeron las mujeres que consultaban por presentar flujo vaginal acompañado de síntomas a nivel vaginal (prurito, hinchazón, dificultad para realizar actividad sexual, enrojecimiento vaginal o placas) y que firmaron el consentimiento informado. Se excluyeron del estudio las pacientes que se encontraban en tratamiento para la infección, aquellas que tuvieron relaciones sexuales 24 horas antes de tomada la muestra y quienes presentaron sangrado menstrual los 10 días previos a la misma.

\section{Muestra}

Se calculó un tamaño de muestra de 230 mujeres por medio del programa STATCALC de Epi Info versión 6, CDC (Centro para el Control de Enfermedades de Atlanta [Estados Unidos]). Para el cálculo de la muestra se revisaron los datos de frecuencia de consulta del centro de salud "La Milagrosa". En total, se reportaron 900 consultas por vaginitis. Se tuvo en cuenta una frecuencia esperada de infecciones vaginales de $40 \%$ y una menos aceptable de $34 \%$ (de acuerdo con datos de la literatura nacional), con un intervalo de confianza de 95\%. El tamaño de muestra mínimo fue de 199 pacientes. Para la selección de la muestra se utilizó un método de selección simple, sistemático y consecutivo durante un periodo de tres meses (noviembre, diciembre de 2007 y enero de 2008), durante el cual se estudiaron todas las mujeres que asistieron a consulta.

\section{Procedimiento}

A cada paciente se le dio previa explicación detallada del estudio. Se realizó una encuesta, para conocer los antecedentes clínicos y personales, en la que se preguntó por la presencia de síntomas tales como prurito, hinchazón vaginal y dificultad para realizar actividad sexual. También se registraron los siguientes signos descritos por la paciente: color del flujo (amarillo, amarillo-verdoso o blanco), hemorragia, enrojecimiento vaginal y olor fétido.

Además de los signos y síntomas, se tuvo en cuenta el número de episodios de infección vaginal que la paciente había sufrido en el último año (presencia de flujo con síntomas), si era VIH positivo, si padecía cáncer, si estaba en embarazo o si había estado bajo tratamiento con corticoides.

Una vez la paciente aceptó participar en el estudio y firmó el consentimiento informado, se procedió a tomar la muestra. Las muestras fueron tomadas de las paredes laterales de la vagina, introduciendo el espéculo estéril sin lubricar. Se utilizó un aplicador con algodón para tomar la muestra de la parte interna de la pared vaginal, procurando que ésta no presentara sangre. Inmediatamente después, se frotó la muestra en un portaobjetos (de forma circular de afuera hacia adentro) y se colocó el escobillón en un tubo de ensayo con $1 \mathrm{ml}$ de solución salina 
estéril. Consecutivamente, se introdujo un segundo escobillón para obtener una muestra de la parte externa de la vagina, la cual se extendió de forma circular en el mismo portaobjetos. Finalmente, se depositó el escobillón en otro tubo con solución salina estéril. La lámina portaobjetos se dejó secar y se guardó en una caja de portaobjetos con el fin de que la muestra no se perdiera para el posterior análisis del fresco y para la observación de los microorganismos mediante la coloración de Gram. Se examinaron la vulva, los labios mayores y menores, y se observaron las características macroscópicas de la secreción vaginal; para lo cual se tuvo en cuenta: color, aspecto y consistencia (homogénea o grumosa). Este procedimiento fue realizado por la auxiliar de laboratorio del centro de salud.

Después de tomar las muestras, tanto los tubos de ensayo como los portaobjetos se guardaron en una nevera de icopor y se transportaron hasta el Centro de Investigaciones Biomédicas de la Universidad del Quindío. Los resultados del análisis de las muestras fueron consignados en un formato de informe del frotis vaginal, que fue entregado como resultado del examen.

En el laboratorio, las muestras fueron incubadas a $37^{\circ} \mathrm{C}$ durante $30 \mathrm{~min}$. Después, se hizo un examen en fresco y una coloración de Gram. Se observaron las muestras al microscopio con aumento de $10 \mathrm{x}$ recorriendo toda la placa, luego con aumento de 40x (esta observación fue realizada por los dos bacteriólogos del laboratorio), y en seguida, con aumento de 100x. Posteriormente se realizó la identificación de bacterias, hongos y protozoos.

El diagnóstico de vaginosis bacteriana se realizó sólo si se presentaban por lo menos tres de los cuatro criterios clínicos propuestos por Amsel (flujo fino, blanco adherente y homogéneo, $\mathrm{pH}$ superior a 4,5, prueba de amina positiva, y presencia de células indicadoras -células clave- en la preparación con solución salina). Todas las pruebas fueron examinadas por dos observadores.

Para la identificación de hongos se realizó un cultivo microbiológico de Sabouraud y se utilizó la prueba de $\mathrm{KOH}$ al 30\%; se consideró positivo la presencia de levaduras, y para Candida albicans, una prueba de tubo germinal positiva. Para la identificación de protozoos (Trichomonas) se hizo un frotis directo luego de incubar la muestra fresca a $37^{\circ} \mathrm{C}$ en solución salina al 0,8\% y se visualizó al microscopio en $40 x$.

\section{Variables a medir}

Para el análisis de los resultados se evaluó la presencia de microorganismos tales como Trichomonas, hongos (pseudomicelios y blastoconidias) y bacterias (cocobacilos gram variable tipo Gardnerella y una variedad de bacterias) en conjunto con ausencia de Lactobacillus o escasa presencia de éstos. Además de estas variables, se tuvo en cuenta: edad, antecedentes de infecciones vaginales, presencia y tipo de sintomatología, antecedentes de infecciones de transmisión sexual, uso de medicamentos, presencia de secreción cervical purulenta, $\mathrm{pH}$, test de aminas y características del flujo.

\section{Análisis}

La base de datos fue elaborada en Excel y analizada con el paquete estadístico Epi Info versión 6. Los datos clínicos y microbiológicos fueron organizados en una hoja de trabajo de Excel para luego ser importados al programa Epi Info versión 6 (CDC, Atlanta). Se presentaron las frecuencias relativas de cada tipo de aislamiento con el respectivo intervalo de confianza, se llevó a cabo un análisis comparativo de las variables estudiadas y se analizó la relación existente entre agente causal, síntomas y signos.

\section{Aspectos éticos}

El comité de ética aprobó la investigación y la calificó como de riesgo mínimo. El estudio se desarrolló como parte de un trabajo de grado para la licenciatura en Biología y de acuerdo con la legislación nacional e internacional para la investigación con muestras de origen humano, en especial con el decreto 8430 de 1993 del Ministerio de Salud. Todos los resultados de los exámenes fueron entregados a las pacientes y no se realizaron exámenes que no buscaran 
beneficio para las mismas. La confidencialidad se mantuvo durante todo el estudio y los resultados sólo fueron entregados por el personal de salud, en sobre cerrado.

\section{RESULTADOS}

\section{Características generales y distribución de microorganismos}

Entre las 230 mujeres incluidas en el estudio, 82\% eran gestantes (190 casos) y 17\% no gestantes (40 casos) con edades comprendidas en un rango de 13 a 45 años. Se encontró una prevalencia de infección vaginal de 90,4\% (208 casos) distribuídos de la siguiente forma: 39\% de origen bacteriano, 6,5\% de origen micótico, 5,7\% de Trichomonas, y 39,3 de infecciones mixtas hongos-bacterias o bacteriasbacterias (tabla 1). Igualmente, se detectó un $\mathrm{pH}$ mayor de 4,5 en el 72\% de la población estudiada.

\section{Características de la vaginosis}

En 32\% de las 90 pacientes con infección bacteriana, ésta fue causada por cocobacilos gram variable tipo Gardnerella (29 casos), seguido por Difteroides en un 30\% (27 casos), 10\% por cocobacilos gramnegativos ( 9 casos), 6,6\% por bacilos gramnegativos (6 casos), 3,3\% por cocobacilos grampositivos (3 casos) y $2 \%$ por Mobiluncus (2 casos). Los síntomas más frecuentes, manifestados en los 29 casos de infecciones bacterianas por cocobacilos gram variable tipo Gardnerella como diagnóstico único, fueron el prurito (48\%) y la presencia de olor fétido (67\%); y el signo más frecuente fue el flujo de color blanco (67\%). El pH vaginal fue mayor a 5,5 en el 71\% de los casos.

\section{Características de la infección por Candida}

Los síntomas asociados principalmente con la infección por Candida en las 15 pacientes con esta infección como diagnóstico único, fueron el prurito (93,3\%), el enrojecimiento vaginal (74\%) y la hinchazón vaginal $(28,6 \%)$. El signo más frecuente fue el flujo de color blanco (59,8\%); mientras que el $\mathrm{pH}$ vaginal fue menor a 4,5 en $56 \%$ de los casos. También se encontró que las mujeres en embarazo manifestaban la mayor prevalencia de vaginitis $(5,7 \%)$ frente a $0,5 \%$ de las mujeres que no estaban en estado de embarazo.

\section{Características de la infección por Trichomonas vaginalis}

Se observó flujo de color blanco en $61,5 \%$ de las pacientes con tricomoniasis y el $\mathrm{pH}$ vaginal estuvo entre 4,5 y 5,5 en $100 \%$. Todos los 13 casos de mujeres con infección vaginal por Trichomonas se encontraban en estado de gestación.

\section{Infección mixta: vaginosis-candidiasis}

En 46 casos (20\% de las infecciones) se detectó infección vaginal por asociaciones entre bacterias

Tabla 1. Distribución etiológica de microorganismos en pacientes con síntomas de vaginitis en el centro de salud "La Milagrosa" de Armenia (Quindío, Colombia) entre los meses de febrero-abril de 2008 ( $\mathrm{n}=230$ ).

\begin{tabular}{|l|c|c|}
\hline & Número de casos & $\%$ \\
\hline Infección de origen bacteriano & 90 & $39 \%$ \\
\hline Infección de origen micótico & 15 & $6,5 \%$ \\
\hline Tricomoniasis & 13 & $5,7 \%$ \\
\hline Infección mixta (hongos-bacterias) & 46 & $20 \%$ \\
\hline Infección mixta (bacterias-bacterias) & 44 & $19,3 \%$ \\
\hline Total & $208 / 230$ & $90,8 \%$ \\
\hline
\end{tabular}


y hongos. De las 46 pacientes con diagnóstico de vaginosis bacteriana-candidiasis, el 30\% presentaron asociación entre hongos y difteroides (14 casos), 23,9\% presentaron asociación entre hongos y bacilos gramnegativos (11 casos), 23,9\% presentaron asociación entre hongos y bacterias tales como Mobiluncus, cocobacilos gramnegativos extracelulares, cocobacilos grampositivos (11 casos) y 21,7\% presentaron asociación entre hongos y cocobacilos gram variable tipo Gardnerella (10 casos).

Los siguientes síntomas y signos se asociaron con este diagnóstico: prurito vulvovaginal en un 18,4\%, flujo de color amarillo en un 14\% y olor fétido en el 9,2\%. El pH vaginal fue de 5,5 en $100 \%$ de los casos.

\section{DISCUSIÓN}

El análisis microbiológico logró identificar una etiología microbiana para 90\% de las pacientes en este estudio y, a su vez, de este porcentaje, la mayoría fue por causa bacteriana. La alta frecuencia de identificación de microorganismos es de esperar en una población que asiste a consulta por estos síntomas, lo cual muestra la importancia de realizar el análisis de laboratorio, ya que las manifestaciones clínicas fueron similares tanto en la etiología por bacterias (vaginosis) como en la causada por hongos (Candida), de las cuales cada una tiene un tratamiento diferente. Por esta razón, el análisis de laboratorio estaría indicado en toda mujer que consulta por esta causa. En el presente estudio no se encontraron diplococos gramnegativos intracelulares y no se realizaron cultivos para caracterizar presencia de $N$. gonorrhoeae, por lo cual no es posible determinar si este germen estaba presente o no; y por lo tanto, esto amerita estudios adicionales enfocados a esta infección. La prevalencia de $N$. gonorrhoeae puede variar desde 0,1\% en Turquía ${ }^{15}$ a 1,2\% en mujeres de regiones rurales en Perú. ${ }^{16}$ De otra parte, no se hicieron pruebas de antígenos para buscar infección por Chlamydia trachomatis.

Entre los estudios realizados en Colombia, el presente trabajo encuentra la prevalencia más alta para Trichomonas. Un estudio previo en trabajadoras sexuales en Montería reportó una frecuencia de 2,9\% de este tipo de infección en trabajadoras sexuales y de $0 \%$ en amas de casa, ${ }^{13}$ aunque de esta última población la muestra fue sólo de 16 mujeres. En otro estudio en Medellín, la frecuencia de infección por Trichomonas fue de 2,8\% en la población de estudiantes universitarias. ${ }^{8}$ Sin embargo, se requiere un estudio de factores de riesgo para enfermedades de transmisión sexual en la población del presente estudio, con el fin de determinar las razones para esta prevalencia y si éstas están relacionadas con características particulares de la población analizada, y así, finalmente, poder proponer programas de promoción y prevención que logren disminuir esta prevalencia.

En el presente estudio, Gardnerella estuvo presente en 39\% de los casos. En estudios previos de población sintomática se reportan prevalencias de Gardnerella de 22,6\% al igual que en México $^{17}$ pero, asimismo, se han encontrado otras mucho más altas, como por ejemplo en Haití donde éstas fueron de $60 \% .{ }^{18}$ Del mismo modo, en otro estudio llevado a cabo en mujeres que se realizaban el examen de citología en Bucaramanga, se encontró una prevalencia de 28,2\%. ${ }^{11}$ En el caso de las gestantes del Hospital Belén de Trujillo (Perú), se observaron prevalencias altas en donde la vaginosis fue de $50 \%$, seguida por la candidiasis con $20 \%$ y la tricomoniasis con $11 \% .{ }^{19}$ Esto confirma la importancia de realizar los estudios epidemiológicos para cada sitio, ya que las diferencias pueden ser importantes de acuerdo con la zona geográfica y el tipo de población estudiada.

Por el contrario, la prevalencia de infección vaginal de origen micótico en el presente estudio fue muy baja $(6,5 \%)$ con respecto a lo reportado en otros estudios en donde se encuentran frecuencias de $19 \%$ en México ${ }^{16}$ o de $12 \%$ en amas de casa en Montería. Llamativamente, en esa misma ciudad, las trabajadoras sexuales sólo presentaron Candida en 2,9\% de los casos estudiados. ${ }^{13}$ Es posible que la presencia de microorganismos como Trichomonas o los causantes de vaginosis 
alteren el microambiente vaginal y disminuyan la frecuencia de Candida.

Las principales fortalezas del estudio son: que la muestra representativa de la población que asiste a consulta en este centro de salud fue analizada bajo criterios estandarizados, y por lo tanto, se logró obtener datos locales sobre la prevalencia en relación a la etiología en una población sintomática; y que los análisis de laboratorio fueron realizados por dos observadores independientes. Por el contrario, la debilidad del estudio se halla en que no se realizaron pruebas para N. gonorrhoeae o Chlamydia trachomatis.

En conclusión, el microorganismo más prevalente en mujeres con infección vaginal fue el cocobacilo gram variable tipo Gardnerella, seguido por la candidiasis. Además, se plantea la necesidad de evaluar la identificación microbiológica en relación al diagnóstico sindromático ya que las características clínicas no permiten hacer un diagnóstico preciso del agente causal de manera que se pueda indicar el tratamiento adecuado en los casos positivos.

\section{AGRADECIMIENTOS}

A la Doctora Vilma Collazos y al personal auxiliar de enfermería del centro de salud "La Milagrosa" de Armenia por su colaboración en la toma de muestras.

\section{REFERENCIAS}

1. Spence D, Mellville C. Vaginal discharge. BMJ 2007; 335:1147-51.

2. Hay P. National guideline for the management of bacterial vaginosis. Clinical Effectiveness Group. British Association for Sexual Health and HIV. 2006. Visitado 2009 Abr 3. Disponible en: http://www.guideline. gov/summary/summary.aspx?doc_id $=11602$

3. Amsel R, Totten PA, Spiegel CA, Chen KC, Eschenbach D, Holmes KK. Nonspecific vaginitis. Diagnostic criteria and microbial and epidemiologic associations. Am J Med 1983;74:14-22.

4. Nugent RP, Krohn MA, Hillier SL. Reliability of diagnosing bacterial vaginosis is improved by a standardized method of gram stain interpretation. J Clin Microbiol 1991;29:297-301.
5. Ferrer J. Vaginal candidosis: epidemiological and etiological factors. Int J Gynaecol Obstet 2000;71:S21-7.

6. Schwebke JR, Burgess D. Trichomoniasis. Clin Microbiol Rev 2004;17:794-803.

7. Monterrosa-Castro A, Blaquicet-Anaya L, CantilloCabarcas J, Muñoz-Marrugo L, Valverde-Farre A. Gardnerella vaginalis in reports of cervico-vaginal cytology. Gac Med Mex 1996;132:119-25.

8. Diaz F, Vasquez ME, Escobar S, Galeano A, Londono M, Pelaez M, et al. Vaginitis due to Gardnerella vaginalis in a university medical service. Acta Med Colomb 1985;10:197-203.

9. Duarte HG, Romero JA, Schmalbach JE. Association between the cervico-vaginal inflammatory cytology and the intraepithelial cervical lesion in patients from a sexual and reproductive health clinic in Bogotá, Colombia 1999-2003. Rev Salud Pública (Bogotá) 2004;6:253-69.

10. Vargas CI, Galindo BL, Martínez JE. Vaginitis y cervicitis en planificación familiar. Rev Colomb Obstet Ginecol 1991;42:223-6.

11. Gamboa EM, Vera LM, Castro M, Camacho L. Infecciones vaginales, condición relevante en el centro de reclusión de mujeres de Bucaramanga. Visitado 2009 Abr 3. Disponible en: http://www.javeriana. edu.co/fcea/convocatorias/memorias_1congreso_sp/ carteles/217w.pdf

12. Ardila J, Gómez J, Angel E. Concordancia entre el diagnóstico clínico y paraclínico de las cervico-vaginitis más frecuentes. Rev Colomb Obstet Ginecol 1996; 47:247-51.

13. Alvis N, Mattar S, Garcia J, Conde E, Diaz A. Sexuallytransmitted infection in a high-risk group from Montería, Colombia. Rev Salud Publica (Bogota) 2007;9:86-96.

14. Tolosa JE, Chaithongwongwatthana S, Daly S, Maw WW, Gaitán H, Lumbiganon P, et al. The International Infections in Pregnancy (IIP) study: variations in the prevalence of bacterial vaginosis and distribution of morphotypes in vaginal smears among pregnant women. Am J Obstet Gynecol 2006;195:1198-204.

15. Ozturk CE, Ozdemir I, Yavuz T, Kaya D, Behcet M. Etiologic agents of cervicovaginitis in Turkish women. Saudi Med J 2006;27:1503-7.

16. García PJ, Chavez S, Feringa B, Chiappe M, Li W, Jansen KU, et al. Reproductive tract infections in rural women from the highlands, jungle, and coastal regions of Peru. Bull World Health Organ 2004;82:483-92. 
17. Flores-Paz R, Rivera-Sánchez R, García-Jiménez E, Arriaga-Alba M. Etiología de la infección cérvico vaginal en pacientes del Hospital Juárez de México. Salud Publica Mex 2003;45:S694-7.

18. Behets FM, Desormeaux J, Joseph D, Adrien M, Coicou G, Dallabetta G, et al. Control of sexually transmitted diseases in Haiti: results and implications of a baseline study among pregnant women living in cite Soleil Shantytowns. J Infect Dis 1995;172:764-71.

19. Medina R, Rechkemmer A, Garcia-Hjarles M. Prevalencia de vaginitis y vaginosis bacteriana en pacientes con flujo vaginal anormal en el Hospital Nacional Arzobispo Loayza. Rev Med Hered 1999; 10:144-50.

Declaración sobre conflictos de interés y fuentes de financiación: los autores del presente trabajo declaran no conocer conflictos de interés sobre los resultados expuestos en este artículo. Los autores no han recibido beneficios por parte de las empresas productoras de los reactivos o materiales utilizados en la presente investigación. Los materiales y reactivos de este trabajo fueron financiados por la Universidad del Quindío. 